\title{
Calibration procedures for a halide lon-Selective Electrode array
}

\author{
M. Baret $^{1, *}$, P. Fabry ${ }^{2}$, D.L. Massart ${ }^{3}$, C. Menardo ${ }^{1}$ and M. Fraysse ${ }^{1}$ \\ ${ }^{1}$ Rhône-Poulenc Industrialisation, CRIT Décines, 24 avenue Jean Jaurès, 69153 Décines Charpieu Cedex, France \\ ${ }^{2}$ L.E.P.M.I., École Nationale Supérieure d'Électrochimie et d'Électrométallurgie de Grenoble, \\ BP. 75, F-38402 Saint Martin d'Hères Cedex, France \\ ${ }^{3}$ Vrije Universiteit Brussel, Laarbeeklaan 103, B-1090 Brussel, Belgium
}

\begin{abstract}
Univariate and multivariate Chemometric methods are compared for the determination of ion concentrations measured with a 6 Ion-Selective Electrode array. Principal component analysis is applied as an exploratory method to observe the extent to which interferences occur. Two univariate calibration methods are compared. It is shown that univariate inverse calibration performs at least as well as classical calibration. The use of the former is therefore recommended. The use of partial least squares, principal component regression and multivariate regression is discussed and the possibilities of the method are described.
\end{abstract}

Key words. Ion-selective electrode - univariate calibration - multivariate calibration - halide.

\section{Introduction}

Only a few articles dealing with both Ion-Selective Electrode (ISE) Arrays and Chemometrics can be found in the literature. Otto et al. [1] showed that an over-determined approach of the problem for multiple ion determinations is feasible with $\mathrm{Ca}^{2+}, \mathrm{Mg}^{2+}, \mathrm{Na}^{+}, \mathrm{K}^{+}$. Beebe et al. [2-3] stated that using an array of sparingly selective electrodes gives better results than using an array of highly selective electrodes (use of non linear multiple regression, for $\mathrm{Na}^{+}, \mathrm{K}^{+}$). Forster et al. [4-5] found that better results are obtained when one sparingly selective electrode is associated with highly selective electrodes to determine a low concentration of one of the cations $\left(\mathrm{Na}^{+}, \mathrm{K}^{+}, \mathrm{Ca}^{2+}\right)$ in a medium where other cations are present in a large amount.

To evaluate the possibility of using ISE arrays and chemometrics we developed an array of 6 ISEs for the determination of some halide ions and we investigated how to set up a calibration procedure for this array. Univariate and multivariate chemometrics methods will be compared for the determination of ion concentrations measured with total or part of the 6 ISE array. Our purpose was to investigate the characteristics of a number of uni- and multivariate calibration methods under circumstances for which characteristics of the sensors are well known. The chosen experimental conditions are such that for most ions, univariate methods investigated should give sufficiently good results. On the other hand, for $\mathrm{Cl}^{-}$, very unsuitable conditions were selected, while for $\mathrm{Br}^{-}$four ISEs are relevant. The questions asked are, for instance, whether an array of four ISEs and multivariate calibration gives better results than a single ISE and univariate calibration, whether inverse calibration is equally good as classical calibration, etc. This study should help to decide more generally when to apply ISE arrays, how to carry out calibration and what difficulties to be expect.

\section{Theory}

\section{Ion-selective electrode theory}

An ISE measures solution potential (see for instance [6]) which is related to the free primary ion activity by the Nernst equation (1), but can also be related to the free interfering ion activity by the Nikolskii relation (2) according to the IUPAC recommendations [7].

$$
\begin{gathered}
E=A+\left(R T / z_{i} F\right) \ln a_{i} \\
E=A+\left(R T / z_{i} F\right) \ln \left[a_{i}+\sum_{j} K_{i, j}\left(a_{j}\right)^{z_{i} / z_{j}}\right]
\end{gathered}
$$

where $E$ is the ISE potential versus the reference electrode, $A$ a constant representing the standardisation potential, $R$ the perfect gas constant, $T$ the temperature, $F$ the Faraday constant, $a_{i}$ and $z_{i}$ are the activity and the charge number of the primary ion, $a_{j}$ and $z_{j}$ the activity and the charge number of the $j$ interfering ions, and $K_{i, j}$ is the interference coefficient.

Assuming the activity coefficient to be equal to 1 (diluted solutions), the ion activity is equal to the ion concentration and the equations (1) and (2) can be respectively written as:

$$
\begin{gathered}
E=A+\left(R T / z_{i} F\right) \ln C_{i} \\
E=A+\left(R T / z_{i} F\right) \ln \left[C_{i}+\sum_{j} K_{i, j}\left(C_{j}\right)^{z_{i} / z_{j}}\right]
\end{gathered}
$$

where $C_{i}$ and $C_{j}$ are the concentration of the primary and interfering ions respectively. 


\section{Original articles}

$R, F$ and $\ln 10$ can be gathered in a $B$ constant and writing $\left(-\log C_{x}\right)$ as $p X$, equations (5) and (6) can also be written:

$$
\begin{gathered}
E=A+B \frac{T}{z_{i}} p X \\
E=A+B \frac{T}{z_{i}} \log \left[C_{i}+\sum_{j} K_{i, j}\left(C_{j}\right)^{z_{i} / z_{j}}\right]
\end{gathered}
$$

Instead of the Nikolskii relation, use of a modified Nernstian law has been described in the literature [8] in the case of ISFET (Ion-Sensitive Field-Effect Transistor) calibration for $\mathrm{H}^{+}, \mathrm{K}^{+}, \mathrm{Na}^{+}$, according to the relation (7):

$$
E_{i}=A+\sum_{j} k_{i j} \log C_{j}
$$

where $k_{i j}$ are constants depending on the solution and the ions $i$ and $j$. Good results were obtained in such a description.

In our paper, $p X$ will be determined from the ISE potentials and a relation similar to relation (7) is assumed to describe the ISE potential.

\section{Chemometrics}

The aim of using chemometrics here is to decide whether non-classical calibration methods might improve the calibration of the ISE array and to predict analyte concentrations of other samples of the same kind.

Firstly a Principal Component Analysis (PCA) was performed to have an overview of the data [9]. The PCA was performed on the whole data set to find what the role of the six variables is and to which extent they interact. At the same time we wanted to see whether PCA may play a role in the evaluation of data of this type. Equation (8) gives the relation between the original variables, the scores and the loadings.

$$
X\left(V^{T}\right)^{-1} S^{-1}=U
$$

where $X$ is the $(n \times p)$ matrix of sensor response: $p$ columns corresponding to the sensors and $n$ rows corresponding to the samples. $V$ is the loading matrix corresponding to the weights of the original variables in the latent variables ( $V^{T}$ is the transposed $V$ matrix), $U$ is the score matrix describing the data in the latent variable axes system, and $S$ is the variance matrix.

In this paper, a capital letter denotes a matrix (e.g. $X$ ), a lower-case letter denotes a vector (e.g. $x$ ), and an italic lower-case letter denotes a scalar (e.g. $x$ ).

Several multivariate modeling methods were applied to find a correlation between the $X$ matrix of the measurement data (here the ISE potential responses) and the $Y$ matrix composed here of $p X$, and to make calibrations. The $Y$ matrix is $(n \times q)$ : the $q$ columns correspond to the analytes and the $n$ rows to the samples.

The multivariate methods are [9]:

- Multivariate Linear Regression (MLR), which is a regression of several or all $x$ vectors of the $X$ matrix on one $y$ variable of the $Y$ matrix. Univariate linear regression will be considered as a special case of MLR.

- Principal Component Regression (PCR), where the number of the $x$ variables is reduced. Since the principal com- ponents are orthogonal, this allows to be free of collinearity between variables.

- Partial Least Squares Regression (PLS1), in which the number of the $x$ variables is also reduced but maximising at the same time covariance between $X$ and $y$.

All these methods build models of the type:

$$
y_{i}=b_{0}+b_{1} x_{1 i}+b_{2} x_{2 i}+\ldots+b_{n} x_{n i}+\varepsilon_{i} .
$$

where the errors $\varepsilon_{i}$ are supposed to be normally and independently distributed in $y$, with a constant variance and mean zero.

Different validation procedures are possible:

- Internal validations i.e. performing many times the calibration on the calibration set by cross-validation such as leave-one-out: calibration is performed $n$ times, removing each time one sample (row) of the matrix and predicting it with the calibration model of the $(n-1)$ calibration samples remaining.

- External validation i.e. a test set different from the calibration set (other samples) is predicted with the calibration model.

In a first step, internal validation (cross-validation) is applied to optimise each multivariate method (i.e. determining how many and which variables should be included in the model). To compare models, the Root Mean Square Error of Cross-Validation (RMSECV) is determined. It is the error on the $y$ variable $(p X)$ of the calibration set predicted by crossvalidation, defined as:

$$
\begin{gathered}
R M S E C V=\sqrt{\frac{1}{n} \sum_{i=1}^{n}\left(\hat{f}_{i j}\right)^{2}} \\
\hat{f}_{i j}=y_{i j}-\hat{y}_{i j}
\end{gathered}
$$

where $n$ is the number of samples in the calibration set, $\hat{f}_{i j}$ are the residuals of the model and $y_{i j}$ and $\hat{y}_{i j}$ are respectively the true and the estimated values of $y$ for the $i^{\text {th }}$ row and the $j^{\text {th }}$ column of the calibration set. $\hat{y}_{i j}$ is predicted with the model built from the $(n-1)$ other samples.

A further validation of the best model is performed using an independent validation set (external validation) and the Root Mean Square Error of Prediction (RMSEP) of the test set is obtained:

$$
\begin{aligned}
\mathrm{RMSEP} & =\sqrt{\frac{1}{m} \sum_{i=1}^{m}\left(\hat{g}_{i j}\right)^{2}} \\
\hat{g}_{i j} & =y_{i j}-\hat{y}_{i j}
\end{aligned}
$$

where $m$ is the number of samples in the test set, $\hat{g}_{i j}$ are the residuals of the model and $y_{i j}$ and $\hat{y}_{i j}$ are respectively the true and the estimated values of $y$ for the $i^{\text {th }}$ row and the $j^{\text {th }}$ column of the test set. $\hat{y}_{i j}$ is predicted with the model built from the $n$ calibration samples. 
A fit of the calibration set by the chosen model is also performed and the Root Mean Square Error of $y$ Calibration (RMSEC) is:

$$
\begin{aligned}
\text { RMSEC } & =\sqrt{\frac{1}{n} \sum_{i=1}^{n}\left(\hat{h}_{i j}\right)^{2}} \\
\hat{h}_{i j} & =y_{i j}-\hat{y}_{i j}
\end{aligned}
$$

where $\hat{h}_{i j}$ are the residuals of the model, $y_{i j}$ and $\hat{y}_{i j}$ are respectively the true and the estimated values of $y$ for the $i$ th row and the $j^{\text {th }}$ column of the calibration set. $\hat{y}_{i j}$ is predicted with the model built from the $n$ calibration samples.

\section{Experimental}

\section{Experimental set up}

Since no complete system consisting of an ISE array and a data acquisition system is commercially available, we built our own system (cf. Fig. 1).

A magnetically stirred cell controlled in temperature was used. The 6 commercial ISEs used are from RadiometerTacussel [10]. The characteristics of these ISEs are given in the table I. Each electrode is connected to a millivoltmeter with one single reference electrode for the whole array. A calomel double junction electrode was used with a $\mathrm{K}_{2} \mathrm{SO}_{4}$ $0.5 \mathrm{M}$ electrolyte solution in the second junction. The millivoltmeters are connected to a multiplexer driven by a computer. Millivoltmeters are also from Radiometer-Tacussel (PHM220 ${ }^{\circledR}$ model), the multiplexer $\left(\mathrm{M} 4 \mathrm{DAC}{ }^{\circledR}\right.$ model) is

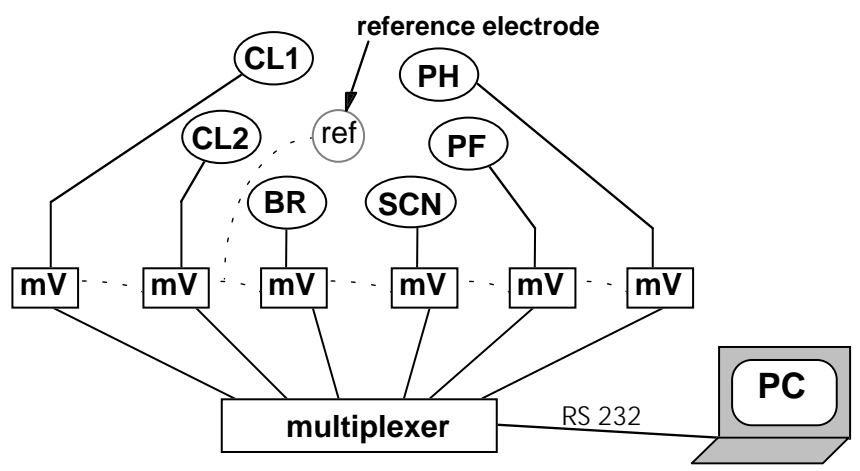

Fig. 1. Experimental set up. " $m V "$ are the millivoltmeters and "PC" is the computer. from BayTec, the computer is a COMPAC prolinea $5100^{\circledR}$ and the software used for data acquisition is home-made.

For the data analysis, we use the Multivariate Calibration Toolbox from ChemoAC ${ }^{\circledR}$ [11], working under Matlab ${ }^{\circledR}$ software [12].

\section{Measurement procedure}

The sample is introduced in the temperature controlled cell. The ISE array is immersed in the solution. After thermal stabilisation of the sample at $25{ }^{\circ} \mathrm{C}$, six measurements are acquired in the following way: when the corresponding ISE

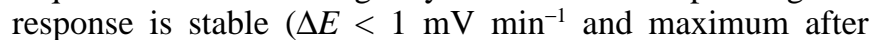
5 min) each millivoltmeter send its potential reading to the multiplexer; when all have responded, the first measurement of the ISE array is recorded and the second measurement is engaged. Only the three last measurements are kept and their average will be used as the ISE array response for the sample.

\section{Samples}

The samples are synthetic aqueous solutions containing $\mathrm{NaCl}, \mathrm{NaBr}, \mathrm{NaF}$ and the electrolyte is $\mathrm{K}_{2} \mathrm{SO}_{4}$ with citric acid and $\mathrm{NaOH}$. The concentration levels have been set up according to a Doehlert experimental design with four factors $(\mathrm{pCl}, \mathrm{pBr}, \mathrm{pF}, \mathrm{pH})$, resulting in 25 calibration solutions including 5 central points. The Doehlert design (Fig. 2) has been chosen, because it presents some advantages: its flexibility allows to move the design in one or another direction if the levels chosen are not the best possible. It is also possible to add a new factor by adding only a few experiments without starting again from the beginning all the experiments [13].

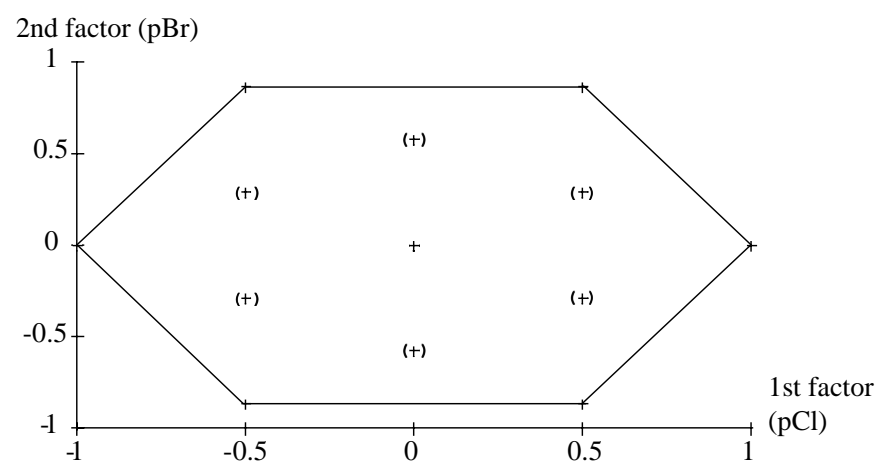

Fig. 2. Doehlert experimental design for 2 factors. (+) correspond to the points added for a design with more than 2 factors.

Table I. Characteristics of commercial ISEs [10]. The highest values of the $p X$ response range correspond to the detection limit.

\begin{tabular}{lcccccc}
\hline ISE name & Reference & Nature & Primary ion & $p X$ response range & Advised $p H$ range & Interference \\
\hline CL1 & XS200 & polycrystalline & $\mathrm{Cl}^{-}$ & $1-6$ & $2-4$ & $\mathrm{~S}^{2-}, \mathrm{CN}^{-}, \mathrm{OH}^{-}, \mathrm{Br}^{-}, \mathrm{I}^{-}$ \\
CL2 & $\mathrm{XS} 210$ & polycrystalline & $\mathrm{Cl}^{-}$ & $1-5$ & $2-14$ & $\mathrm{~S}^{2-}, \mathrm{CN}^{-}, \mathrm{OH}^{-}, \mathrm{Br}^{-}, \mathrm{I}^{-}$ \\
BR & $\mathrm{XS} 22$ & polycrystalline & $\mathrm{Br}^{-}$ & $1-6$ & $2-14$ & $\mathrm{~S}^{2-}, \mathrm{CN}^{-}, \mathrm{OH}^{-}, \mathrm{Cl}^{-}, \mathrm{I}^{-}$ \\
PF & $\mathrm{XS} 270$ & monocrystalline & $\mathrm{F}^{-}$ & $1-6$ & $0-8$ & $\mathrm{OH}^{-}$ \\
PH & $\mathrm{XG} 200$ & glass & $\mathrm{H}^{+}$ & $1-14$ & $0-14$ & \\
SCN & $\mathrm{XS} 250$ & polycrystalline & $\mathrm{SCN}^{-}$ & $1-6$ & $0-14$ & $\mathrm{~S}^{2-}, \mathrm{CN}^{-}, \mathrm{OH}^{-}, \mathrm{Br}^{-}, \mathrm{Cl}^{-}, \mathrm{I}^{-}$ \\
\hline
\end{tabular}




\section{Original articles}

Table II shows the $p X$ and the concentrations corresponding to the design. For reasons of practical opportunity in sample preparation, the $\mathrm{pCl}$ and $\mathrm{pBr}$ levels are not exactly the ones expected when using the theoretical design levels. For the validation, we build another sample set according to the same theoretical Doehlert experimental design but with more restricted $p X$ ranges (cf. Tab. III), so that the test set is fully included in the calibration range.

\section{Results and discussion}

Some ISE properties are known and generally given by the constructor but it is not evident to build a good calibration model on this basis because, although interference coefficients can be approximately known for some ISEs, they can depend on the ionic strength and on the ionic species in the solution [14]. In an industrial application, these can vary and it will be better to be free from such problems.

As already explained, the ISE array and the concentration levels were chosen such that some questions concerning univariate and multivariate calibration can be answered. The ISE array was constructed as follows. To study ISE responses to $\mathrm{Br}^{-}$and $\mathrm{Cl}^{-}$ions, the electrodes have been selected so that four of them (CL1, CL2, BR, SCN) should respond to both ions. The conditions are such that all these electrodes should respond mainly to $\mathrm{Br}^{-}$, with perhaps slight interferences of $\mathrm{Cl}^{-}$because the membrane of the four electrodes are based on silver salts and the theoretical interference coefficient between $\mathrm{Cl}^{-}$and $\mathrm{Br}^{-}$results in their salt solubility ratio (about $10^{2}$ ). This means that the $\mathrm{Cl}^{-}$ concentration must be $10^{2}$ times greater to the $\mathrm{Br}^{-}$one to be taken into account in the ISE potential. The conditions are very unfavourable for $\mathrm{Cl}^{-}$because except for the sample number 6 , the $\mathrm{Br}^{-}$concentration will be the most important in relation (6). It is to be expected that calibration for $\mathrm{Cl}^{-}$ will prove to be very bad. The fluoride ISE (PF) is known to be very selective responding only to free $\mathrm{F}^{-}$ion and to $\mathrm{OH}^{-}$when $\mathrm{pH}$ is higher than 8 . In the studied samples, $\mathrm{pH}$ of 8 is never reach but it is probable that there will be a slight interference of $\mathrm{OH}^{-}$on the $\mathrm{F}^{-}$electrode response. A $\mathrm{pH}$ electrode $(\mathrm{PH})$ which theoretically does not respond to any other investigated ion is also added to the array and $\mathrm{pH}$ is added as a variable in the experimental design. The array is then composed with both very selective and sparingly selective electrodes.

\section{Principal component analysis}

A principal component analysis of the calibration measurement data shows that four principal components (PC)

Table II. Experimental design for the calibration: $p X$ values.

\begin{tabular}{lllllllllllllll}
\hline Sample & 1 & 2 & 3 & 4 & 5 & 6 & 7 & 8 & 9 & 10 & 11 & 12 & 13 & 14 \\
\hline $\mathrm{pCl}$ & 2 & 3 & 1 & 1.3 & 2.3 & 1.3 & 2 & 2.3 & 1.3 & 2.3 & 1.3 & 2 & 2 & 2.3 \\
$\mathrm{pBr}$ & 2.5 & 2.5 & 2.5 & 1 & 4 & 4 & 2.5 & 1 & 2 & 3 & 3 & 1.5 & 2.5 & 2 \\
$\mathrm{pF}$ & 3 & 3 & 3 & 3 & 3 & 3 & 3 & 3 & 2 & 4 & 4 & 4 & 3 & 2 \\
$\mathrm{pH}$ & 6 & 6 & 6 & 6 & 6 & 6 & 6 & 6 & 6 & 6 & 6 & 6 & 6 & 6 \\
& & & & & & & & & & & & & \\
\hline Sample & 15 & 16 & 17 & 18 & 19 & 20 & 21 & 22 & 23 & 24 & 25 & \\
\hline $\mathrm{pCl}$ & 2 & 1.3 & 2.3 & 1.3 & 2 & 2 & 2 & 2.3 & 2 & 2 & 2 & \\
$\mathrm{pBr}$ & 3.5 & 2 & 3 & 3 & 2.5 & 1.5 & 2.5 & 2 & 3.5 & 2.5 & 2.5 & \\
$\mathrm{pF}$ & 2 & 2.75 & 3.25 & 3.25 & 3 & 3.25 & 2.25 & 2.75 & 2.75 & 3.75 & 3 & \\
$\mathrm{pH}$ & 6 & 7 & 5 & 5 & 6 & 5 & 5 & 7 & 7 & 7 & 6 & \\
\hline
\end{tabular}

Table III. Experimental design for the validation: $p X$ values.

\begin{tabular}{lllllllllllllll}
\hline Sample & 1 & 2 & 3 & 4 & 5 & 6 & 7 & 8 & 9 & 10 & 11 & 12 & 13 & 14 \\
\hline $\mathrm{pCl}$ & 2 & 2.8 & 1.2 & 1.6 & 2.4 & 1.6 & 2 & 2.4 & 1.6 & 2.4 & 1.6 & 2 & 2 & 2.4 \\
$\mathrm{pBr}$ & 2.5 & 2.5 & 2.5 & 1.2 & 3.8 & 3.8 & 2.5 & 1.2 & 2.175 & 2.825 & 2.825 & 1.525 & 2.5 & 2.175 \\
$\mathrm{pF}$ & 3 & 3 & 3 & 3 & 3 & 3 & 3 & 3 & 2.2 & 3.8 & 3.8 & 3.8 & 3 & 2.2 \\
$\mathrm{pH}$ & 6 & 6 & 6 & 6 & 6 & 6 & 6 & 6 & 6 & 6 & 6 & 6 & 6 & 6 \\
& & & & & & & & & & & & \\
\hline Sample & 15 & 16 & 17 & 18 & 19 & 20 & 21 & 22 & 23 & 24 & 25 & \\
\hline $\mathrm{pCl}$ & 2 & 1.6 & 2.4 & 1.6 & 2 & 2 & 2 & 2.4 & 2 & 2 & 2 & \\
$\mathrm{pBr}$ & 3.475 & 2.175 & 2.825 & 2.825 & 2.5 & 1.525 & 2.5 & 2.175 & 3.475 & 2.5 & 2.5 & \\
$\mathrm{pF}$ & 2.2 & 2.8 & 3.2 & 3.2 & 3 & 3.2 & 2.4 & 2.8 & 2.8 & 3.6 & 3 & \\
$\mathrm{pH}$ & 6 & 6.8 & 5.2 & 5.2 & 6 & 5.2 & 5.2 & 6.8 & 6.8 & 6.8 & 6 & \\
\hline
\end{tabular}


describe $98.4 \%$ of the total variance (Tab. IV). The first one describes $54.4 \%$ of the variance and the loadings show that this PC represents a combination of the CL1, CL2, BR and SCN electrode responses with almost the same influence. The second PC describes $21.9 \%$ of the variance and represents PH electrode variations. PC3 explains $19.0 \%$ of the variance and represents $\mathrm{PF}$ electrode response with a slight effect of $\mathrm{pH}$, and $\mathrm{PC} 4$ describes only $3.1 \%$ of the total variance and represents responses of the four first electrodes with a largest influence of the CL1 electrode. In figure 3 which describes the loadings, CL1 has a positive influence on PC4 and SCN, BR and CL2 have a negative influence. This means that PC4 probably describes a combination of $\mathrm{pCl}$ and $\mathrm{pBr}$ and probably the $\mathrm{Cl}^{-} / \mathrm{Br}^{-}$interactions, which are different for CL1 and CL2.

The score plots (Fig. 4) show that the repeatability of the measurements is quite good because, the points representing the samples $1,7,13,19$ and 25 are very close together. It can be noticed in figure $4 \mathrm{a}$ that the score plot of the calibration set looks like the biased Doehlert experimental design if $\mathrm{pH}$ is represented versus $\mathrm{pBr}$ (Fig. 5). This can be understood if PC1 essentially represents $\mathrm{pBr}$ and $\mathrm{PC} 2$ represents $\mathrm{pH}$. In these score plots, there is no evident outlier except perhaps sample 6 (cf. Fig. 4b). The slight shift of sample 6 versus lower $\mathrm{PC} 1$, representing $\mathrm{pBr}$, can be explained by the high ratio between chloride and bromide concentrations for this sample. In figure $4 b$, the seven $\mathrm{pF}$ levels can be distinguished on the PC3 axis but there is no obvious relation between $\mathrm{pCl}$ or $\mathrm{pBr}$ levels and $\mathrm{PC} 4$.
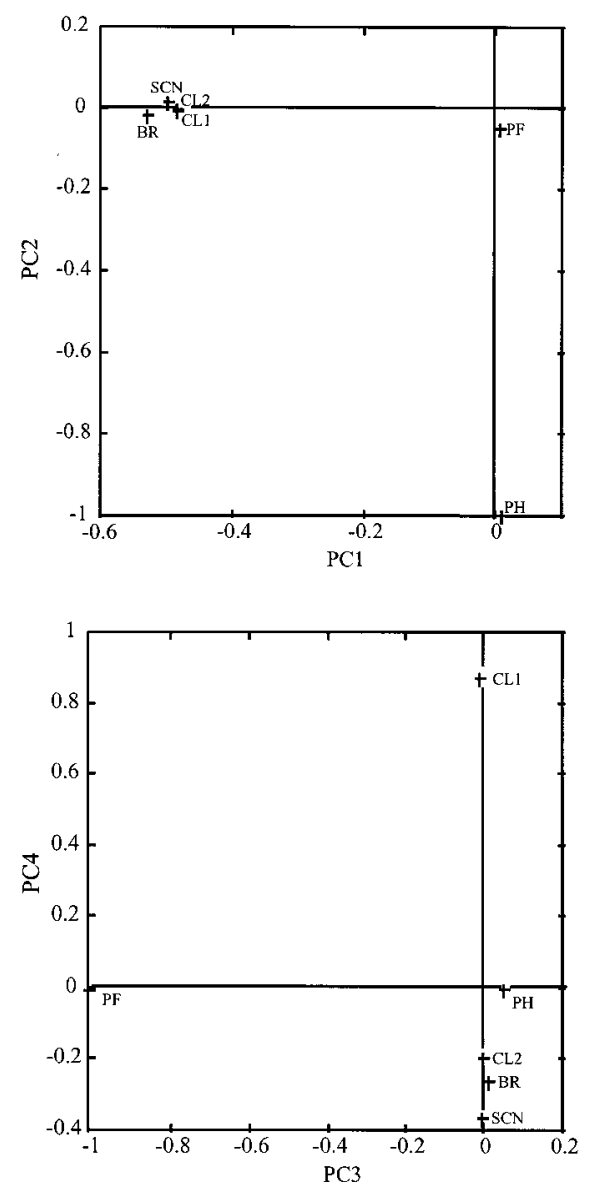

Table IV. PCA, \% of explained variance.

\begin{tabular}{lcc}
\hline $\begin{array}{l}\text { Principal } \\
\text { componant }\end{array}$ & $\begin{array}{c}\text { \% of explained } \\
\text { variance }\end{array}$ & $\begin{array}{c}\text { total variance } \\
\text { explained }(\%)\end{array}$ \\
\hline PC1 & 54.4 & 54.4 \\
PC2 & 21.9 & 76.3 \\
PC3 & 19.0 & 95.3 \\
PC4 & 3.1 & 98.4 \\
\hline
\end{tabular}

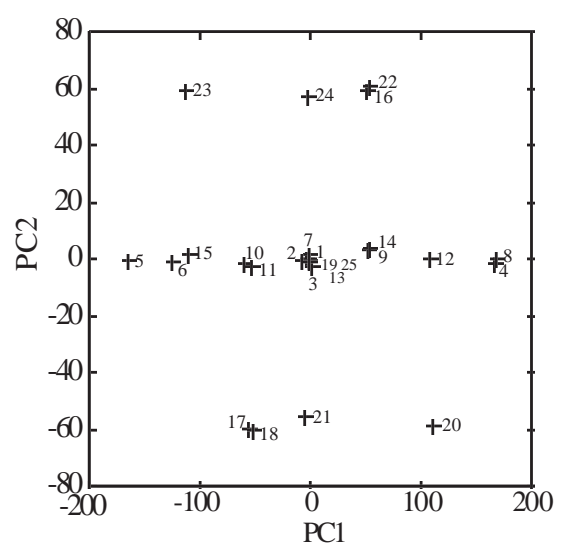

Fig. 4a.

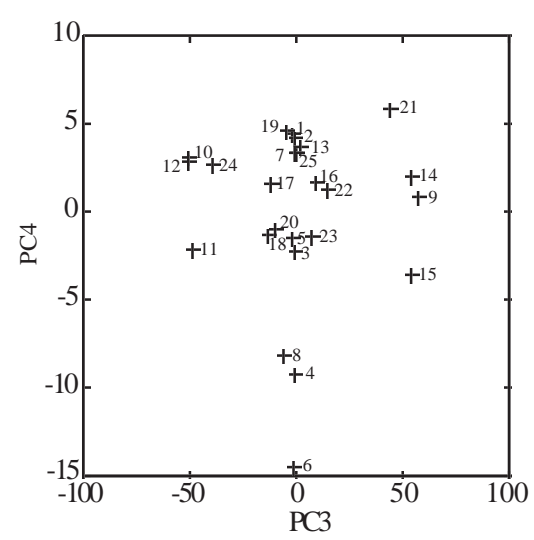

Fig. $4 \mathrm{~b}$

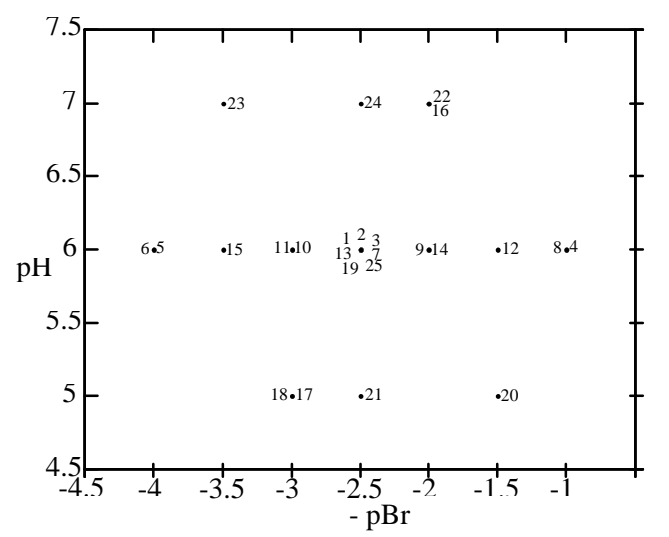


In figure 6 each $\mathrm{PC}$ is represented versus the corresponding pX. In figure $6 \mathrm{a}$, sample 6 is not as the other samples on the straight line. It confirms what was theoretically expected for this sample with the Nikolskii relation (relation (2)). Indeed, the interference coefficient $\mathrm{K}_{\mathrm{Br}^{-}, \mathrm{Cl}^{-}}$of chlorides on the bromide selective electrode and the $\mathrm{Br}^{-}$and $\mathrm{Cl}^{-}$concentrations for this sample are such that the chloride term in relation (2) will be more important than the bromide one, which is not the case for the other samples. It is immediately clear that there is no electrode measuring preferentially the $\mathrm{pCl}$ variations in the studied samples and it is expected that it will not be possible to model $\mathrm{pCl}$. Nevertheless, some

Fig. 6a.

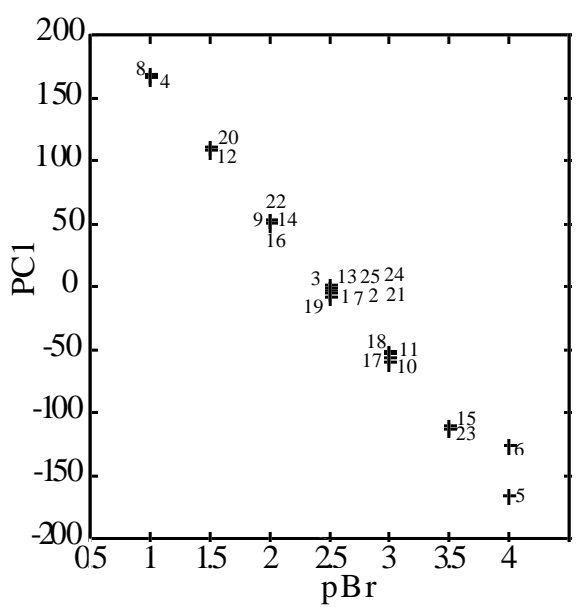

Fig. 6b.

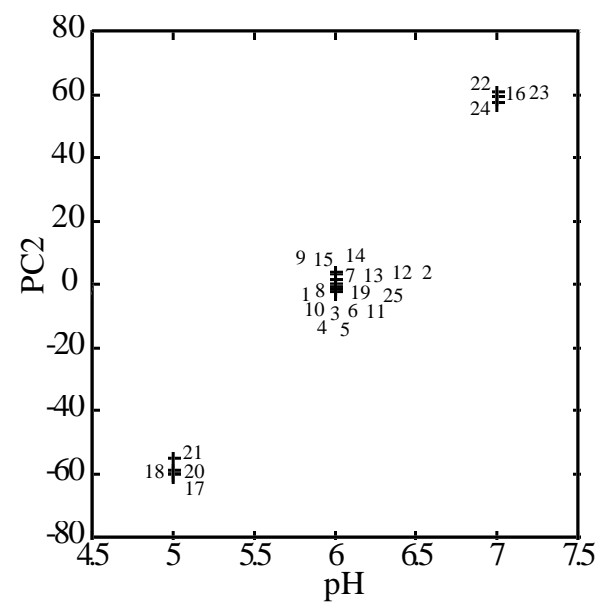

Fig. 6c.

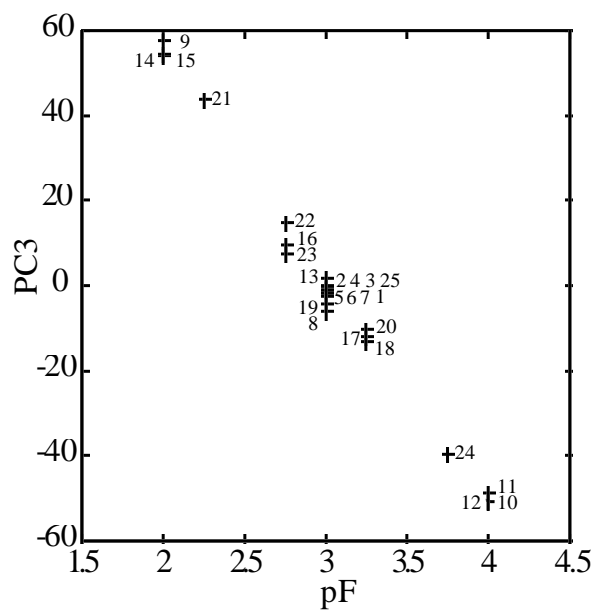

chloride information could be present as interferences of $\mathrm{Cl}^{-}$ on bromide estimation with a model using CL1 electrode like it could be the case in PC4.

Figure 6c shows a non linearity between $\mathrm{pF}$ and PC3. This could be explained by a slight influence of $\mathrm{pH}$ on this PC (Fig. 3). A test of significance (F-test) shows that there is a lack of fit to a first degree model and not to a second degree model (for a second degree model, $p=0.0019$, $F($ exp. $)=1.977$ and $F(\mathrm{tab})=2.927)$.

We can conclude that a principal component analysis indeed allows to see the possibilities and the difficulties to be expected, namely the probable impossibility to analyse $\mathrm{Cl}^{-}$, the slight interference of $\mathrm{Cl}^{-}$on $\mathrm{Br}^{-}$for CL1 ISE and of $\mathrm{pH}\left(\mathrm{OH}^{-}\right)$on $\mathrm{F}^{-}$for PF ISE in a certain range.

\section{Calibrations}

Multivariate calibration methods can be applied when several sensors contribute to the measurement of a substance. These methods (MLR, PCR, PLS, neural nets) have received much attention, mainly in spectroscopy. All these methods show the peculiarity that they carry out inverse calibration, i.e. the model is written as:

$$
\begin{gathered}
\text { concentration or } p X= \\
f \text { (signal, e.g. potential or absorbance) }
\end{gathered}
$$

while classical calibration is carried out according to the equation:

$$
\text { signal }=f(\text { concentration or } p X) .
$$

There is a statistical reason to carry out univariate calibration according to equation (17), namely that in these applications one usually knows the concentration of the standards accurately, while the measurement signal is subject to (normally distributed) measurement error. When ordinary least squares regression (OLS) is applied, the squared residuals of the $y$-values (measurement values) towards the regression line are minimised. It appears at first sight not to be logical to minimise them in the $x$-direction (concentrations or $p X$ ) since they should be known without error. In the usual multivariate calibration application (e.g. with NIR applications) the situation is different. One takes real samples, which means that the concentrations are not known and have to be determined with a reference method, so that there are now errors in both the measurements and the $p X$. There is no statistical reason to use equation (17) anymore and, for other reasons which are too long to explain here, equation (16) is preferred.

In the present case, there is a dilemma. On the one hand, we would like to apply multivariate calibration, because as follows from the PCA study, some sensors are not absolutely selective in the studied range. On the other hand, this means that we would have to apply equation (16), although we are in a situation where concentrations are theoretically known, so that one would rather prefer to apply equation (17) but inverse calibration has a sense even though there is no error on the concentration because errors are transposed. Another interest of the inverse calibration is that when the problem is ill-conditioned, the results are better than the ones obtained by direct method. In this article, we investigate this dilemma. First we compare the application of equations (16) and (17) for the univariate situation. 


\section{Univariate results}

By linear regression of each ISE response on $\mathrm{pBr}$, it appears that, as was expected, four electrodes (BR, CL1, CL2 and $\mathrm{SCN})$ respond to bromides. The highest correlation coefficient is found for the CL2 electrode, and the two other ISEs ( $\mathrm{PF}$ and $\mathrm{PH}$ ) respond linearly to their primary ion. On the other hand, all the univariate regressions on $\mathrm{pCl}$ are very bad (correlation coefficients are lower than 0.06), so that no electrode responds selectively to chlorides in such ranges of concentration. Table $\mathrm{V}$ shows the results obtained by normal and inverse univariate calibration. The results of both methods are very similar but inverse regression often leads to slightly better results in prediction (RMSEP) than the normal one. The plots of the inverse univariate calibrations are shown in figure 7 .
Table V. Inverse and normal univariate calibration results. RMSECV is the root mean square error of leave-one-out Cross-validation on the calibration set, RMSEC is the root mean square error of fit of the calibration set, RMSEP is the root mean square error of prediction of the test set.

\begin{tabular}{lcccccc}
\hline & & \multicolumn{3}{c}{$\begin{array}{c}\text { inverse univariate } \\
\text { calibration }\end{array}$} & $\begin{array}{c}\text { normal univariate } \\
\text { calibration }\end{array}$ \\
\cline { 3 - 7 }$p X$ & $I S E$ & RMSECV & RMSEC & RMSEP & RMSEC & RMSEP \\
\hline $\mathrm{pH}$ & $\mathrm{PH}$ & 0.021 & 0.0198 & 0.0204 & 0.0198 & 0.0205 \\
$\mathrm{pF}$ & $\mathrm{PF}$ & 0.056 & 0.0509 & 0.0427 & 0.0511 & 0.0447 \\
$\mathrm{pBr}$ & $\mathrm{CL} 1$ & 0.1618 & 0.1448 & 0.1564 & 0.1474 & 0.1505 \\
$\mathrm{pBr}$ & $\mathrm{CL} 2$ & 0.0674 & 0.0579 & 0.0453 & 0.0581 & 0.0465 \\
$\mathrm{pBr}$ & $\mathrm{BR}$ & 0.0681 & 0.0592 & 0.0375 & 0.0594 & 0.038 \\
$\mathrm{pBr}$ & $\mathrm{SCN}$ & 0.0749 & 0.0651 & 0.0839 & 0.0654 & 0.0837 \\
\hline
\end{tabular}

Fig. 7a.
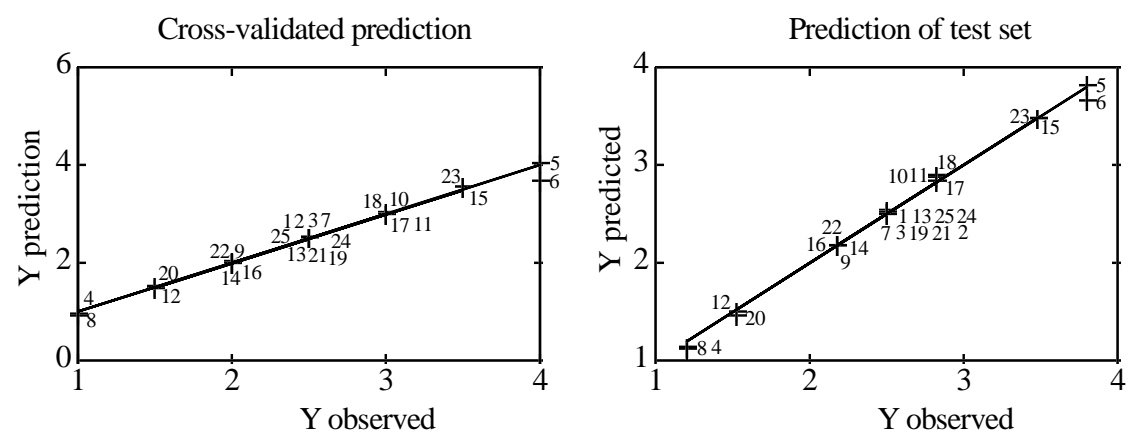

Fig. 7b.
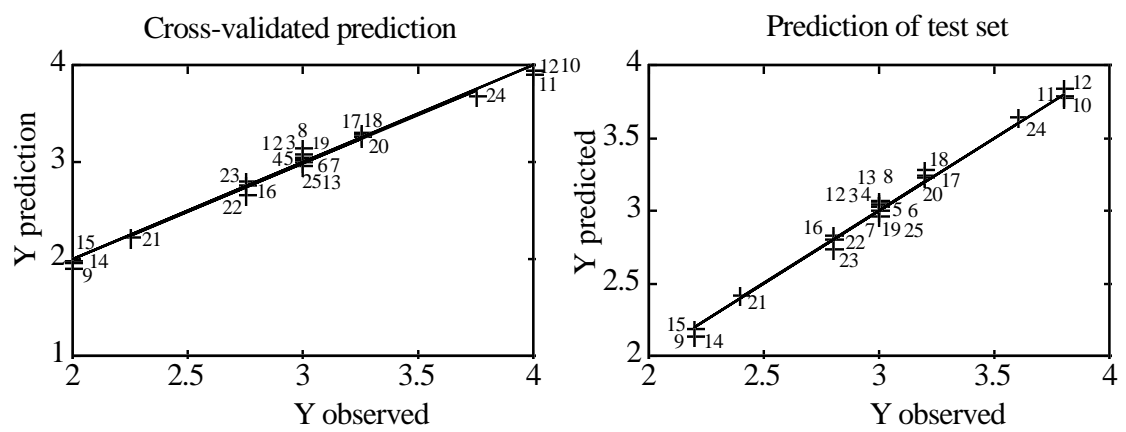

Fig. 7c.
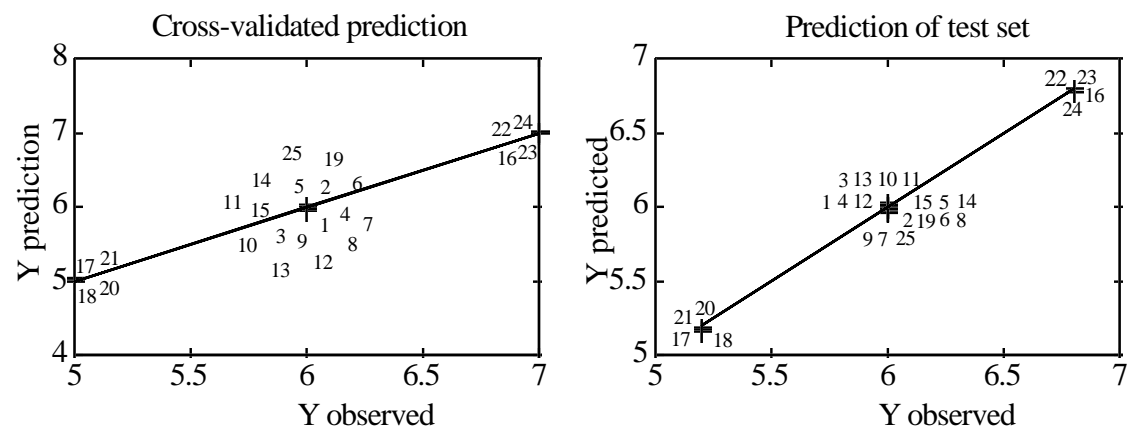

Fig. 7. Univariate inverse calibration results. a) $\mathrm{pBr}$ inverse univariate calibration with CL2 electrode, RMSEP of the test set is $0.0465(1.86 \%)$, b) $\mathrm{pF}$ inverse univariate calibration with $\mathrm{PF}$ electrode, RMSEP of the test set is $0.0447(1.49 \%)$, c) $\mathrm{pH}$ inverse univariate calibration with PH electrode, RMSEP of the test set is $0.0205(0.34 \%)$.
Investigation of the theory learns that in inverse calibration, the $b_{0}$ and $b_{1}$ values are biased [15-18]. The bias depends on

$$
\lambda^{2}=\frac{\sigma\left(\varepsilon_{a}\right)^{2}}{\sigma(\alpha)^{2}}
$$

i.e. depends on the precision of the measurements $\sigma\left(\varepsilon_{a}\right)^{2}$ compared with the range of the measurements, $\sigma(\alpha)^{2}$. The theory $[19,20]$ also shows that this bias is applied in the prediction such that in fact prediction should be better. The somewhat unexpected conclusion is that inverse calibration should always be better than the normally used calibration. The difference depends on $\lambda^{2}$. The standard deviation in the measurement of the 5 central points of the experimental design is used as a measure of repeatability to obtain $\sigma\left(\varepsilon_{a}\right)^{2}$ [19]. As this is small in this case compared to $s(a)^{2}, \lambda$ is small and the improvement should also be small. In fact, in the present case the improvement is expected to be negligible from a practical point of view, but it seemed of interest to verify the theory anyway.

Results in table $\mathrm{V}$ confirms that in all cases inverse calibration should give at least as good results as the classical calibration and that the difference between both approaches seems to be practically negligible. Concerning this part of the study, we can conclude that the 


\section{Original articles}

theory of measurement error in univariate calibration is applicable to these ISE array data and gives results that agree with those obtained from the developed calibration models. The precision of the measurements is good and then it does not really matter whether classical or inverse calibration is used but it is expected that in general inverse calibration using OLS should be the best choice. This will be more important when $\lambda$ is small. While in general the precision of the measurement with ISE is quite good and therefore $\sigma\left(\varepsilon_{a}\right)^{2}$ is small, it can occur in practice that the range of calibration $\sigma(\alpha)$ will not be as large as here, e.g. when the calibration is based on production samples.

\section{Multivariate results}

A second point of interest is whether it is possible to improve the results using multivariate calibration. It is often stated that the use of multivariate calibration gives better results among others because by using different sensors one has an averaging effect. Moreover, it should be possible to correct for interferences. In this study interferences are very limited (except $\mathrm{Br}^{-}$ones onto $\mathrm{Cl}^{-}$) and we are more interested in the averaging effect. The use of different ISEs together in the multivariate methods will result in a combination in the latent variables of the responses of the ISEs, which should produce an averaging effect and the error in the model is expected to be reduced. Since PLS is the most frequently applied chemometric method, we have chosen to focus on PLS. Our aim is to study its performance and also to understand how the method functions in the present context.

\section{pBr PLS models}

In a first step we will study the PLS solution using all sensors. In figure 8a the RMSEP curve for cross-validation of the calibration samples is shown. Normally such curves show first a decrease in RMSEP with number of components included in the PLS model. The number of components yielding the lowest RMSEP or the number of components where RMSEP levels off is selected as optimal. In the present case, however, an increase is found for the second and third PLS-component and one would select the 4-component model, as from this number on the decrease in RMSEP is slight. The anomalous behaviour of the curve, i.e. the intermediate maximum, is due to the criterion that PLS uses. PLS minimises covariance between the $y$ and the $X$ data, i.e. respectively the $\mathrm{pBr}$ and the responses of the six ISEs. The usual RMSEP curve shows a decreasing trend until a minimum is reached, because the correlation part of the covariance dominates. Because the inclusion of the successive components in the PLS model leads to better correlation between $y$ and $X$, a better RMSEP is then obtained. However, here it is the variance part of the covariance which is important and leads to the inclusion of the second and third components. These components essentially include information on the $\mathrm{pH}$ and $\mathrm{pF}$ responses which are irrelevant for the measurement of $\mathrm{pBr}$ but explain much variance in the data. The inclusion of noise due to the irrelevant information in the second and third components leads to an increase in the RMSEP. The RMSEP curve for the test set data has a more usual shape (Fig. 8b). Based on this curve one would select the 1-component PLS model as optimal. If one were to use the 4-component PLS model, the prediction would be somewhat worse than with the 1-component model

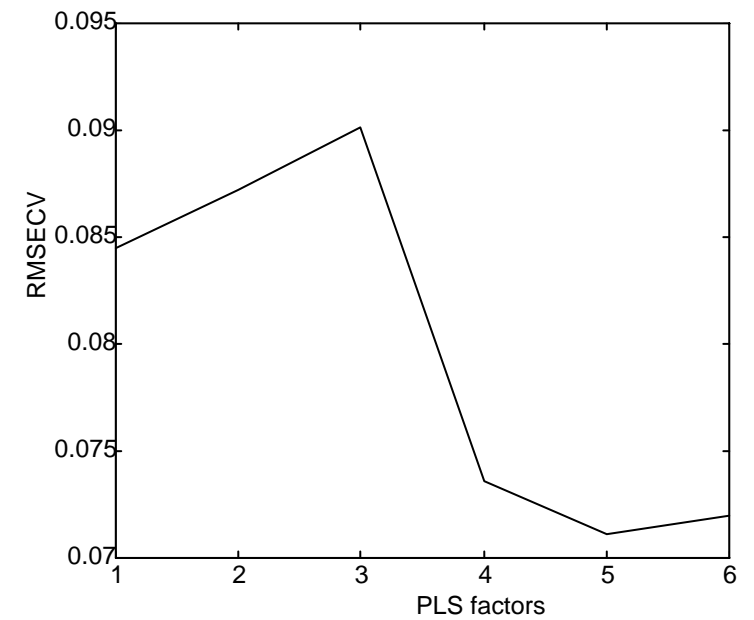

Fig. 8a.

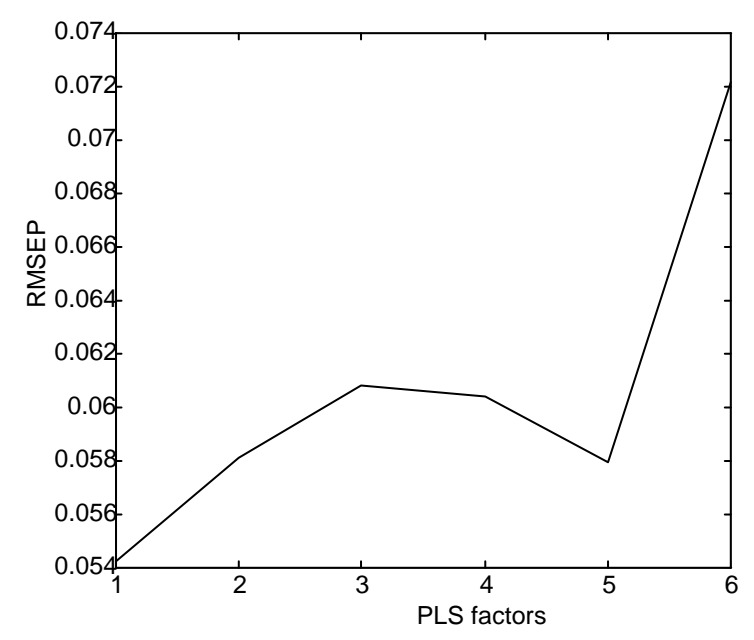

Fig. 8b.

Fig. 8. RMSEP curves for $\mathrm{pBr}$ determination by PLS using 6 ISEs. a) RMSECV curve: cross-validation of the calibration sample set, b) RMSEP curve: prediction of the test sample set.

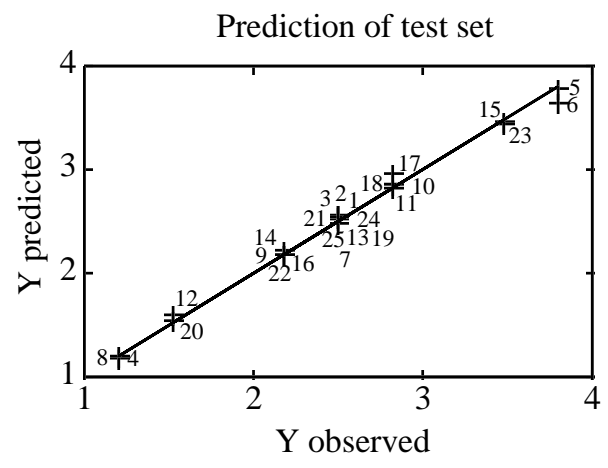

Fig. 9. $\mathrm{pBr}$ predicted for the test set with 1 PLS component against known $\mathrm{pBr}$ value.

(0.060 against 0.054$)$. The plot of concentration predicted against known concentration obtained with the 1-component model for the test set is given in figure 9.

In a second step we will study what happens when we eliminate the $\mathrm{PH}$ and $\mathrm{PF}$ electrodes, thereby retaining only those ISEs that are relevant. It is often stated that the 
inclusion of irrelevant sensors in PLS has no influence on the quality of the prediction: they should receive low weights in the final solution and therefore have little or no influence. On the other hand one would intuitively expect that the inclusion of irrelevant measurement results would increase noise and therefore lead to less good results. The RMSEP curves for the PLS models with the BR, CL1, CL2 and SCN ISE are given in figure 10. One observes that indeed the intermediate maximum in the RMSEP curve for the calibration samples has disappeared. One would now retain a 2-factor model on the basis of cross-validation and again a 1-factor model on the basis of the test set. It is to be noted that the RMSECV for the 2-factor solution for the calibration samples of the model developed for 4 ISE is very similar to that for the 4-component solution for 6 ISE (respectively, 0.0727 and 0.0736). The same is true for the RMSEP of both 1-component models on the test set $(0.0549$ versus 0.0542 ). It is concluded that, at least on the test set, PLS is able to extract the proper information when irrelevant information is present. In the present preliminary study, where it is known exactly what information is important and what not, this is not really important, but it is encouraging for situations where this is not the case. The postulated averaging effect occurs, but it is not positive. The RMSEP of the four individual electrodes is $0.0375,0.0453,0.084$ and 0.156 (Tab. V) and the RMSEP of the four together is 0.0542. PLS is not affected very much by the bad performance of the SCN and CL1 electrodes, but the set of four together is certainly not better than the best univariate electrode.

One can notice that in the shown results, some RMSECV have a lower value than the RMSEP one. This is partly due to the low repeatability of the measurement with an ISE in a mixture containing interferent ions.

\section{UVE-PLS}

Incidentally, we have wondered whether it would be possible to eliminate irrelevant sensors on the basis of an algorithm. In the present preliminary study, we know which sensors are important. In more difficult situations this is less evident and it is interesting to have this type of information. We have therefore evaluated the UVE-PLS algorithm (Uninformative Variable Elimination by PLS) [21]. The UVE-PLS algorithm is applied to eliminate variables which are uninformative, and contribute only noise for the determination considered. If all variables are eliminated, this means that calibration is not possible. This is a very useful diagnostic. Indeed, multivariate calibration is time consuming and a diagnostic which makes it clear from the beginning that calibration will not be possible can save much work. The application of the UVE-PLS algorithm confirms what was experimentally observed concerning the variables which should be used to model $p X: \mathrm{pCl}$ cannot be modeled, for $\mathrm{pBr}$ only $\mathrm{CL} 1, \mathrm{CL} 2, \mathrm{BR}$ and $\mathrm{SCN}$ are relevant, for $\mathrm{pF}$ only $\mathrm{PF}$ and for $\mathrm{pH}$ only PH. Again, it does not seem that the postulated positive averaging effect occurs. The best set of two electrodes (CL2 and BR) yields 1-factor PLS models for both calibration and test samples with RMSEP for the test samples of 0.0344 (and therefore better than for the models with 4 and 6 ISE). In fact, this set of 2 individually best electrodes yields a PLS model which is even somewhat better than the univariate calibration. When we go to the models for inverse calibration for a single ISE, which can be considered as the PLS model for one ISE, we find that

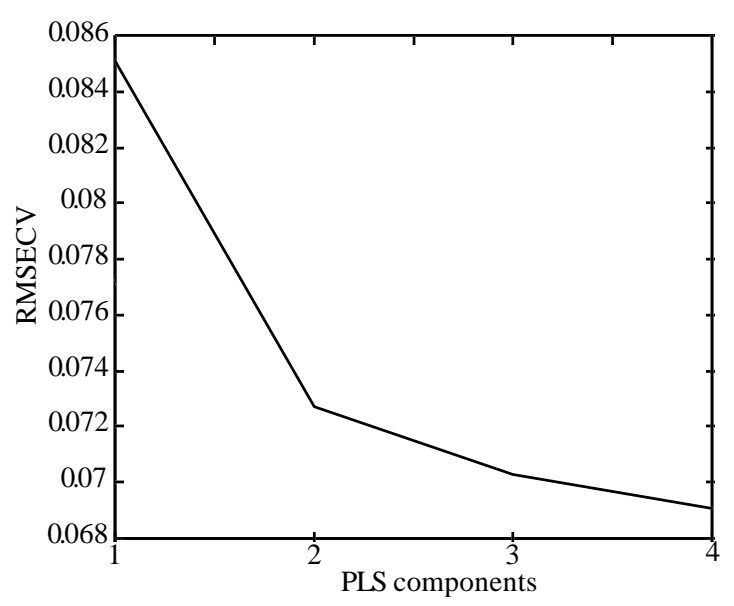

Fig. 10a.

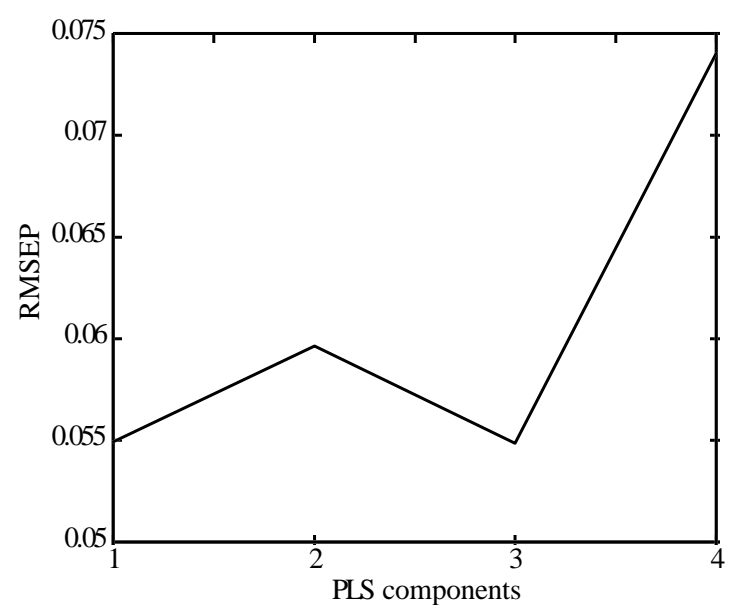

Fig. 10b.

Fig. 10. RMSEP curves for $\mathrm{pBr}$ determination by PLS using BR, CL1, CL2, SCN electrodes. a) RMSECV curve: cross-validation of the calibration sample set, b) RMSEP curve: prediction of the test sample set.

the individual electrodes have RMSEP for the test set of 0.037 for BR to 0.045 for CL2. It is therefore concluded that the use of different sensors together does not improve the univariate calibration to a large extent in such cases.

\section{Other models}

The application of PCR does not yield better results than PLS (see Tab. VI). The PCR solution for the set of all 6 ISE together yields an RMSEP for $\mathrm{Br}^{-}$of 0.0624 , which is very comparable to PLS. The optimal PCR model is based, as could be expected, on PC1 and PC4, since PC2 and PC3 concern information about $\mathrm{F}^{-}$and $\mathrm{pH}$. For the $\mathrm{pF}$ determination we also investigated whether multivariate methods yield good results. The best PCR model is based on PC3 and PC2 and yields an RMSEP of 0.0408. This seems a logical model since PC3 concerns $\mathrm{F}^{-}$information and PC2 concerns $\mathrm{H}^{+}$, which, as described interferes to a small extent. It should be noted however (see Fig. 11) that the inclusion of PC2 has only a small effect, and it is not evident that it is statistically significant. The PCR model does seem slightly better than the univariate model (RMSEP $=0.0427)$. The PLS model contains three components, which is larger than 


\section{Original articles}

Table VI. Multivariate best results. PCR + PC sel. means that principal components are selected minimising the RMSECV. The original or latent variables used to build the model are described in «factors» column.

\begin{tabular}{|c|c|c|c|c|c|c|}
\hline$y$ & ISE array & method & factors & RMSECV & RMSEC & RMSEP \\
\hline $\mathrm{pBr}$ & All & PLS & 4 factors & 0.0736 & 0.0434 & 0.0604 \\
\hline $\mathrm{pBr}$ & All & PLS & 1 factor & 0.084 & 0.074 & 0.0542 \\
\hline $\mathrm{pBr}$ & CL1, CL2, BR, SCN & PLS & 2 factors & 0.0727 & 0.4422 & 0.0597 \\
\hline $\mathrm{pBr}$ & CL1, CL2, BR, SCN & PLS & 1 factor & 0.085 & 0.0740 & 0.0549 \\
\hline $\mathrm{pBr}$ & BR, CL2 & PLS & 1 factor & 0.0663 & 0.0572 & 0.0344 \\
\hline $\mathrm{pBr}$ & All & PCR & $\mathrm{PC} 1, \mathrm{PC} 4$ & 0.0715 & 0.0446 & 0.0624 \\
\hline $\mathrm{pF}$ & All & PCR & $\mathrm{PC} 3, \mathrm{PC} 2$ & 0.0628 & 0.0494 & 0.0408 \\
\hline $\mathrm{pF}$ & All & PLS & 3 factors & 0.0604 & 0.0491 & 0.0406 \\
\hline $\mathrm{pH}$ & All & PLS & 3 factors & 0.0201 & 0.0168 & 0.01917 \\
\hline $\mathrm{pH}$ & All & PCR & $\mathrm{PC} 2, \mathrm{PC} 3$ & 0.0346 & 0.0176 & 0.0196 \\
\hline $\mathrm{pCl}$ & CL1 & MLR & CL1 & 0.497 & 0.457 & 0.369 \\
\hline $\mathrm{pCl}$ & All & PCR & PC4 & 0.458 & 0.414 & 0.4095 \\
\hline $\mathrm{pCl}$ & All & PCR & $\mathrm{PC} 1$ & 0.4977 & 0.4585 & 0.3704 \\
\hline $\mathrm{pCl}$ & All & PLS & 1 factor & 0.5203 & 0.4581 & 0.3702 \\
\hline $\mathrm{pCl}$ & All & PLS & 2 factors & 0.557 & 0.444 & 0.3585 \\
\hline
\end{tabular}

expected at first sight, but can be explained in the same way as the four component model for pBr. MLR selects only one component and a complementary study shows that $\mathrm{pH}$ influence on the $\mathrm{pF}$ determination is not detectable in this data set, nor on the $\mathrm{pBr}$ determination. The measurement of $\mathrm{pH}$ shouldn't be improved by PLS or PCR and that is not the case. It turns out, however, that it is not worse either. The RMSEP of the univariate method, PLS and PCR are comparable.

Concerning $\mathrm{pCl}$ determination, results by univariate calibration were very bad (no correlation between each ISE and the $\mathrm{pCl}$ levels) and we investigated if multivariate calibration methods could solve the problem. Some results are shown in table VI using the different methods and various ISE arrays (we also try to input as original variable the $\mathrm{pBr}$ levels) but the calibration does not exist and in each case the correlation coefficients are very bad.

\section{Conclusion}

A comparison between univariate and multivariate calibration for this example shows that neither multivariate nor univariate calibrations lead to an acceptable result when interferences are very high, as for the $\mathrm{Cl}^{-}$determination in the chosen concentration ranges. When interferences are very small or negligible as for the $\mathrm{pH}$ determination, univariate calibration leads to results comparable to those of the multivariate method. When interferences are not negligible but not too large, like for the bromide determination, multivariate calibration is better than univariate calibration. In the concentration ranges of this work, and because measurement precision is good, there are no important differences between classical or inverse univariate calibration, but, as expected from theory inverse calibration is slightly better.

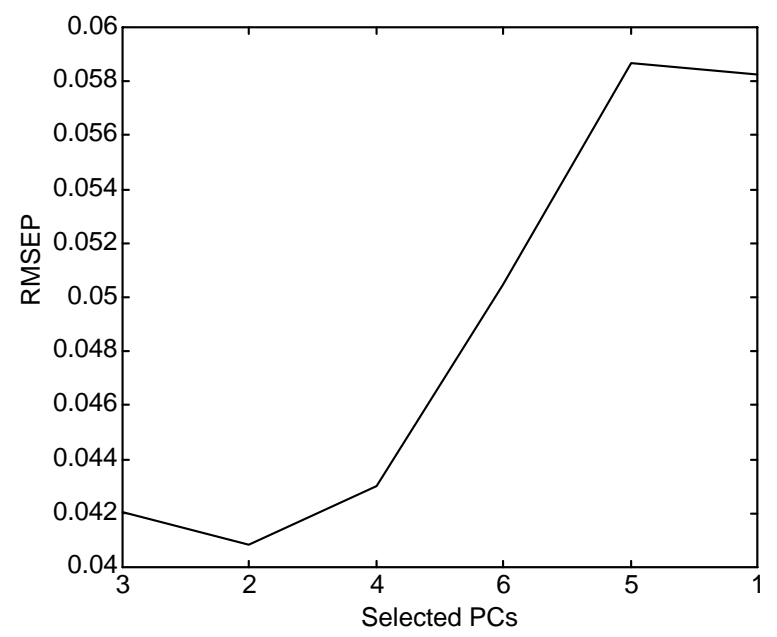

Fig. 11. RMSEP curve for $\mathrm{pF}$ determination by PCR using 6 ISEs.

From these results, it is expected that in case of higher interferences of $\mathrm{Cl}^{-}$onto $\mathrm{Br}^{-}$(and lower ones of $\mathrm{Br}^{-}$onto $\left.\mathrm{Cl}^{-}\right), \mathrm{pCl}$ and $\mathrm{pBr}$ will be calibrated using multivariate methods.

\section{Acknowledgements}

We are very grateful to G. Vallet from Rhône-Poulenc Industrialisation who made the data acquisition software and to D. Jouan-Rimbaud, V. Centner, Q. Guo and S. Kuttatharmmakul from the Vrije Universiteit Brussel (VUB) for their precious help. M. Baret also thanks J.R. Desmurs, G. Albert, F. Conesa, and C. Sterna from Rhône-Poulenc for 
their support and the Standard Measurement and Testing project (CEE) for financial support. D.L. Massart thanks the FWO for financial support.

\section{References}

1. Otto, M.; Thomas, J. R. D. Anal. Chem. 1985, 57, 2647-2651.

2. Beebe, K.; Uerz, D., Sandifer, J.; Kowalski, B. Anal. Chem. 1988, 60, 66-71.

3. Beebe, K.; Uerz, D.; Kowalski, B. Anal. Chem. 1988, 60, 2273-2278.

4. Forster, R.; Regan, F.; Diamond, D. Anal. Chem. 1991, 63, 879-882.

5. Forster, R.; Diamond, D. Anal. Chem. 1992, 64, 1721-1728.

6. Morf, W. E. The principle of ion-selective electrodes and of membrane transport, Elsevier, Amsterdam, 1981.

7. IUPAC Pure Appl. Chem. 1976, 48, 127-132.

8. Igarashi, I.; Ito, T.; Taguchi, T.; Tabata, O.; Inagaki, H. Sensors Actuat. 1990, B1, 8-11.

9. Martens, H.; Naes, T. Multivariate Calibration, John Wiley \& Sons, 1989, ISBN 047190979.

10. Radiometer-Tacussel, Monobloc Ion selective electrodes compacted polycrystalline or mixed crystal model of electrodes, Instruction manual, March 1993, reference DB12U113.
11. Jouan-Rimbaud, D.; Centner, V.; Massart, D. L. A toolbox for multivariate calibration, ChemoAC, Pharmaceutical Institute, Vrije Universiteit Brussel, September 1996.

12. MATLAB 4.0 High-Performance Numeric Computation Software, The MATH WORKS Inc.

13. Feneuille, D.; Mathieu, D.; Phan-Tan-Luu, R. Méthodologie de la Recherche Expérimentale: étude des surfaces de réponse, Document Universitaire, Laboratoire de Prospectives Réactionnelles et d'Analyses de l'Information, Université d'Aix en Provence, January 1983.

14. Cretin, M. Réalisation et étude de capteurs potentiométriques à membranes céramiques sélectives à l'ion lithium. Thèse de doctorat en science, Institut National Polytechnique de Grenoble, 1996.

15. Draper,N. R.; Smith, H. Applied Regression Analysis 2nd ed., Wiley, New-York, 1981.

16. Fuller, W. A. Measurement Error models, Wiley, New-York, 1987.

17. Mandel, J. J. Qual. Technol. 1984, 16, 1-14.

18. Modges, S. D.; Moore, P. G. Appl. Stat. 1972, 21, 185-195.

19. Esbensen, K.; Schonkopf, S.; Midtgaar, T. Multivariate Analysis in Practice, Camo AS, 1994, Trondheim.

20. Centner, V.; de Jong, J.; Massart, D. L. personal communication.

21. Centner, V.; Massart, D. L.; de Noord, O.; de Jong, S.; Vandeginste, B.; Sterna, C. Anal. Chem. 1996, 68, 3851-3858. 